\title{
EXISTENCE AND UNIQUENESS OF SOLUTIONS OF BOUNDARY VALUE PROBLEMS FOR TWO DIMENSIONAL SYSTEMS OF NONLINEAR DIFFERENTIAL EQUATIONS ${ }^{1}$ )
}

\author{
BY \\ PAUL WALTMAN
}

\begin{abstract}
The paper considers the nonlinear system $x^{\prime}=f(t, x, y), y^{\prime}=g(t, x, y)$ with linear and nonlinear two point boundary conditions. With a Lipschitz condition, an interval of uniqueness for linear boundary conditions is determined using a comparison theorem. A corresponding existence theorem is established. Under the assumption of uniqueness, a general existence theorem is established for quite general nonlinearities in the functions and in the boundary conditions. Examples are provided. The results extend previous work on second order scalar differential equations.
\end{abstract}

Introduction. Questions of the existence and uniqueness of solutions of the two point boundary value problem

$$
\begin{gathered}
y^{\prime \prime}+f\left(t, y, y^{\prime}\right)=0, \\
y(a)=A, \\
y(b)=B,
\end{gathered}
$$

where $f\left(t, y, y^{\prime}\right)$ satisfies a Lipschitz condition, have a long history, going back to Picard [9], 1893. The problem is to determine, in terms of the Lipschitz constants, the best possible interval $[a, b]$ on which there exists a unique solution of (1.1), (1.2), (1.3). Recent results and references on this problem can be found in [2], [3]. A second question of interest is, given that solutions of $(1.1),(1.2),(1.3)$ are unique, i.e. given that there exists at most one solution, when does this imply that there exists at least one solution. The first results along this line are due to Lasota and Opial [6] and Jackson [3].

This paper investigates these two types of questions for the second order system

$$
x^{\prime}=f_{1}(t, x, y), \quad y^{\prime}=f_{2}(t, x, y)
$$

subject to more general boundary conditions

$$
\begin{aligned}
& g_{1}(x(a), y(a))=c_{1}, \\
& g_{2}(x(b), y(b))=c_{2} .
\end{aligned}
$$

Received by the editors March 10, 1969 and, in revised form, February 11, 1970.

AMS 1970 subject classifications. Primary 34B15.

Key words and phrases. Nonlinear differential equations, boundary value problem, comparison theorem, existence and uniqueness.

(1) Research supported by NSF Grant GP-8173 and by ONR Contract DAAFO 3-69C-0014.

Copyright (C) 1971, American Mathematical Society 
In $\S 2$ the Lipschitzian problem is treated and in $\$ 3$ the question of proving existence given uniqueness is explored. The theorems are illustrated in $\$ 4$. When a Lipschitz condition is assumed, as in [2], we take it to be of the form

$$
\begin{array}{cl}
L_{1}\left(x_{1}-x_{2}\right) \leqq f_{1}\left(t, x_{1}, y\right)-f_{1}\left(t, x_{2}, y\right) \leqq L_{2}\left(x_{1}-x_{2}\right) & \text { if } x_{1} \geqq x_{2}, \\
K_{1}\left(y_{1}-y_{2}\right) \leqq f_{1}\left(t, x, y_{1}\right)-f_{1}\left(t, x, y_{2}\right) \leqq K_{2}\left(y_{1}-y_{2}\right) & \text { if } y_{1} \geqq y_{2}, \\
M_{1}\left(x_{1}-x_{2}\right) \leqq f_{2}\left(t, x_{1}, y\right)-f_{2}\left(t, x_{2}, y\right) \leqq M_{2}\left(x_{1}-x_{2}\right) & \text { if } x_{1} \geqq x_{2}, \\
N_{1}\left(y_{1}-y_{2}\right) \leqq f_{2}\left(t, x, y_{1}\right)-f_{2}\left(t, x, y_{2}\right) \leqq N_{2}\left(y_{1}-y_{2}\right) & \text { if } y_{1} \geqq y_{2} .
\end{array}
$$

If $f_{i}(t, x, y), i=1,2$, are continuous on $[a, b] \times R \times R$ and satisfy (1.7), solutions of the initial value problem are unique and can be continued over $[a, b]$. The advantage of using (1.7) instead of the usual Lipschitz condition with absolute value is that the added information in (1.7) can lead to sharper estimates on the allowable size of $b-a$.

Many of the arguments used in this paper take place in the $x-y$ plane. By making the substitution $x=\rho \cos \theta, y=\rho \sin \theta$, one obtains a new set of differential equations for $\rho$ and $\theta$. The functions $\theta(t)$, the polar angle, and $\rho(t)$, the polar radius, play an important role.

2. Existence and uniqueness of solutions for Lipschitzian problems. Consider the boundary value problem

$$
\begin{aligned}
& x^{\prime}=f_{1}(t, x, y), \quad y^{\prime}=f_{2}(t, x, y), \\
& A x(a)+B y(a)=c_{1}, \\
& C x(b)+D y(b)=c_{2}, \quad b>a,
\end{aligned}
$$

where the $f_{i}(t, x, y)$ satisfy a Lipschitz condition of the form (1.7). Theorem 2.1 deals with the problem of finding the best possible bound on the size of $b-a$, given the Lipschitz constants, for there to exist at most one solution. An existence theorem for this case is given as Theorem 2.2. The conditions (2.2) and (2.3) can be replaced by a class of nonlinear boundary conditions and it is shown that Theorem 2.1 can be extended to this case at the expense of increased computational complexity.

A principal tool in the approach here will be a comparison theorem of Perov [5, p. 177] (see also [8]). Solutions of (2.1) will be compared with those of an auxiliary system

$$
x^{\prime}=f_{1}(t, x, y), \quad y^{\prime}=\tilde{f}_{2}(t, x, y),
$$

where the $\tilde{f}_{1}(t, x, y), i=1,2$, will be constructed from the Lipschitz constants. First of all, define $\left\{f_{1}, f_{2}\right\} \geqq\left\{\tilde{f}_{1}, \tilde{f}_{2}\right\}$ in a region $D$ if the following inequality is satisfied there.

$$
x f_{2}(t, x, y)-y f_{1}(t, x, y) \geqq x \tilde{f}_{2}(t, x, y)-y \tilde{f}_{1}(t, x, y), \quad(t, x, y) \in D .
$$


Let $\varphi(t)$ be the polar angle (see Introduction) for $(x(t), y(t))$ a solution of (2.1); $\tilde{\varphi}(t)$, the polar angle for $(\tilde{x}(t), \tilde{y}(t))$, a solution of (2.4).

Perov Comparison Theorem. Suppose $(x(t), y(t))$ and $(\tilde{x}(t), \tilde{y}(t))$ exist over $[a, b]$, neither vector being zero there. Let $D=[a, b] \times R \times R$. Suppose $\tilde{f}_{i}(t, c x, c y)=$ $c f_{i}(t, x, y), c>0, i=1,2$, and suppose that solutions of the initial value problem for (2.4) are unique. If $\left\{f_{1}, f_{2}\right\} \geqq\left\{\tilde{f}_{1}, \tilde{f}_{2}\right\}$ and $\varphi(a) \geqq \tilde{\varphi}(a)$, then $\varphi(t) \geqq \tilde{\varphi}(t), t \in[a, b]$. The inequalities may be reversed throughout.

First, define

$$
\begin{aligned}
P_{1}(u, v)=L_{1} u+K_{1} v & \text { if } u v \geqq 0, \\
=L_{2} u+K_{1} v & \text { if } u v<0 ; \\
P_{2}(u, v)=M_{2} u+N_{2} v & \text { if } u v \geqq 0, \\
=M_{2} u+N_{1} v & \text { if } u v<0 ; \\
Q_{1}(w, z)=L_{2} w+K_{2} z & \text { if } w z \geqq 0, \\
=L_{1} w+K_{2} z & \text { if } w z<0 ; \\
Q_{2}(w, z)=M_{1} w+N_{1} z & \text { if } w z \geqq 0, \\
=M_{1} w+N_{2} z & \text { if } w z<0 .
\end{aligned}
$$

The comparison system will be

$$
u^{\prime}=P_{1}(u, v), \quad v^{\prime}=P_{2}(u, v)
$$

and

$$
w^{\prime}=Q_{1}(w, z), \quad z^{\prime}=Q_{2}(w, z) .
$$

The systems (2.6) and (2.7) can have a discontinuity in the right-hand side at a change of quadrant. If differentiability of solutions is not required at these points (left and right derivatives exist but are unequal), solutions exist and are uniquely defined by their initial conditions. Within a quadrant, the right-hand sides are linear and solutions may be computed explicitly.

Let $y=\alpha x$ and $y=\beta x$ be two lines in the $x-y$ plane. Define $p(\alpha, \beta)$ to be the time it takes for a solution of (2.6) which begins at $t=0$ at a point on $y=\alpha x$ to first reach a point on the line $y=\beta x$ (if a solution does reach it) or to be $+\infty$ if no solution beginning on $y=\alpha x$ at $t=0$ reaches $y=\beta x$ for any $t \geqq 0$. Since (2.6) is piecewise linear and homogeneous, $p(\alpha, \beta)$ is independent of the choice of the initial condition on $y=\alpha x$ and of the initial "time" $t=0$. This definition can be interpreted in terms of the polar angle $\omega(t)$ of $(2.6)$, i.e. if $\omega(0)=\alpha, \pi / 2 \geqq \alpha \geqq-\pi / 2$, and $\omega(b)=\beta(\bmod \pi)$, $\pi / 2 \geqq \beta \geqq-\pi / 2$, where $b$ is the first nonnegative time that $\omega(t)=\beta(\bmod \pi)$, then $p(\alpha, \beta)=b$. Since the equation has piecewise constant coefficients, $p(\alpha, \beta)$ can be determined explicitly if needed. Define $q(\alpha, \beta)$ in the same way for the system (2.7). Note that $p(\alpha, \alpha)=q(\alpha, \alpha)=0$. 
THEOREM 2.1. Let $f_{i}(t, x, y), i=1,2$, be continuous on $[a, b] \times R \times R$ and satisfy (1.7) there. If $b-a<M$, where

$$
M=\min \left[p\left(\tan ^{-1}-A / B, \tan ^{-1}-C / D\right), q\left(\tan ^{-1}-A / B, \tan ^{-1}-C / D\right)\right],
$$

there is at most one solution of the boundary value problem (2.1), (2.2), (2.3). This result is best possible.

Proof. Suppose there were two solutions $\left(x_{1}(t), y_{1}(t)\right),\left(x_{2}(t), y_{2}(t)\right)$ of (2.1), (2.2), (2.3). Let $\bar{x}(t)=x_{2}(t)-x_{1}(t)$ and $\bar{y}(t)=y_{2}(t)-y_{1}(t)$ and suppose labeling is chosen so that $\bar{x}(a)>0$ or if $\bar{x}(a)=0, \bar{y}(a)>0$. Such a choice is possible since $(\bar{x}(a), \bar{y}(a)) \neq$ $(0,0)$ by uniqueness of solutions of initial value problems. Then $(\bar{x}(t), \bar{y}(t))$ satisfies the following:

$$
\begin{aligned}
x^{\prime}=F_{1}(t, x, y), \quad y^{\prime} & =F_{2}(t, x, y), \\
A x(a)+B y(a) & =0, \\
C x(b)+D y(b) & =0,
\end{aligned}
$$

where $F_{i}(t, x, y)=f_{i}\left(t, x+x_{1}(t), y+y_{1}(t)\right)-f_{i}\left(t, x_{1}(t), y_{1}(t)\right), i=1,2$. Note that the $F_{i}(t, x, y)$ satisfy the same Lipschitz condition (1.7) as the $f_{i}(t, x, y)$.

Let $(u(t), v(t))$ and $(w(t), z(t))$ be solutions of (2.6) and (2.7) with initial conditions $(u(a), v(a))=(w(a), z(a))=(\bar{x}(a), \bar{y}(a))$. Let $\varphi(t), \omega(t), \psi(t)$ denote the polar angles of $(\bar{x}(t), \bar{y}(t)),(u(t), v(t))$ and $(w(t), z(t))$, respectively. $P_{1}, P_{2}, Q_{1}, Q_{2}$ are positive homogeneous and one verifies that $\left\{P_{1}, P_{2}\right\} \geqq\left\{F_{1}, F_{2}\right\} \geqq\left\{Q_{1}, Q_{2}\right\}$. Since $\varphi(a)=\omega(a)=\psi(a)$ it follows from the Perov Comparison Theorem that $\omega(t) \geqq$ $\varphi(t) \geqq \psi(t)$ and in particular $\omega(b) \geqq \varphi(b) \geqq \psi(b)$.

Now, $b-a<M$ says that no solution of (2.6) or (2.7) which begins on a line through the origin with slope $\tan ^{-1}-A / B$ at $t=a$ can reach a line through the origin with slope $\tan ^{-1}-C / D$ at or before $t=b$. The comparison theorem says that if neither $\omega(t)$ or $\psi(t)$ crosses a line with slope $\tan ^{-1} C / D$, then neither does a solution of (2.8), (2.9). Hence $(\bar{x}(b), \bar{y}(b))$ cannot satisfy $(2.10)$ unless $(\bar{x}(t), \bar{y}(t))=$ $(0,0)$. Such is not the case and this contradiction establishes the theorem.

The result is "best possible" since if $b-a=M$, either (2.6) or (2.7) has a nontrivial solution satisfying (2.9) and (2.10) as well as the trivial solution.

If the lines given by (2.2) and (2.3) are parallel, then $M=0$ and Theorem 2.1 does not apply. That the theorem is not true in this case may be seen from the problem $y^{\prime \prime}+y^{\prime}=0, y^{\prime}(a)=y^{\prime}(b)=0$ which has any constant as a solution. In the important special case $x(a)=c_{1}, x(b)=c_{2}$ this can be remedied by assuming $K_{1}>0$ (which is always the case for second order equations). Then it can be shown that at $\theta= \pm \pi / 2$, $\theta^{\prime}(t)<0$. That is, solutions cross $\theta= \pm \pi / 2$ moving counterclockwise in the plane so that nontrivial solutions of (2.8) can move from $x(a)=0$ to $x(b)=0$ only by passing through an elapsed angle of $\pi$. (For $y(a)=c_{1}, y(b)=c_{2}$ this can be remedied by assuming $M_{2}<0$.) Then the comparison equations can be used to determine an interval so small that $\theta(t)$ cannot pass through such an angle. 
The existence theorem corresponding to Theorem 2.1 is

THEOREM 2.2. If the hypotheses of Theorem 2.1 hold, then there exists a solution of (2.1), (2.2), (2.3).

Proof. Let $\left(x_{1}(t), y_{1}(t)\right)$ be an arbitrary solution of (2.1), (2.2). If (2.3) is not satisfied, then either $C x_{1}(b)+D y_{1}(b)=\delta<c_{2}$ or $>c_{2}$. We suppose the former; a similar argument applies in the second case. The idea of the proof is to find initial conditions for a solution which lies on the opposite side of the line $C x+D y=$ $c_{2}$. This will be done using (2.8) and (2.9). In particular, we seek a solution $(r(t), s(t))$ of (2.8), (2.9) with $C r(b)+D s(b)<c_{2}-\delta$, so that $\left(x_{2}(t), y_{2}(t)\right)=$ $\left(r(t)+x_{1}(t), s(t)+y_{1}(t)\right)$ satisfies $(2.1),(2.2)$ and $C x_{2}(b)+D x_{2}(b)>c_{2}$.

Let $(u(t), v(t)),(w(t), z(t))$ be solutions of (2.6), (2.7) with polar angles $\omega(t), \psi(t)$, with $\omega(a)=\psi(a)=\tan ^{-1}-A / B$. The rays $\omega(b)$ and $\psi(b)$ are not parallel to $C x+$ $D y=0$ so $\omega(b)$ and $\psi(b)$ intersect the line $C x+D y=c_{2}$ at points whose radii in polar coordinates we label $R_{\omega}$ and $R_{\psi}$. Let $R=\max \left[R_{\omega}, R_{\psi}\right]$. We need the following very elementary lemma.

LEMMA. Let $\rho(t)$ be the polar radius of (2.8). Then $\rho(t)$ satisfies

$$
\begin{gathered}
\rho(a) e^{-\sigma(t-a)} \leqq \rho(t) \leqq \rho(a) e^{\sigma(t-a)}, \\
a \leqq t \leqq b, \quad \sigma=\sum_{i=1}^{2}\left(\left|K_{i}\right|+\left|L_{i}\right|+\left|M_{i}\right|+\left|N_{i}\right|\right) .
\end{gathered}
$$

Proof of lemma. The equation for $r(t)$ is

$$
r^{\prime}=F_{1}(t, r \cos \theta, r \sin \theta) \cos \theta+F_{2}(t, r \cos \theta, r \sin \theta) \sin \theta .
$$

Using the Lipschitz condition, (1.7), it foilows that $\sigma r \geqq r^{\prime} \geqq-\sigma r$ and an integration establishes the lemma.

Proof of theorem (continued). Choose an initial radius $\rho(a)$ such that $\rho(a)>$ $R e^{\sigma|b-a|}$. Let $(r(t), s(t))$ be a solution of (2.8), (2.9) such that $r^{2}(a)+s^{2}(a) \geqq \rho^{2}(a)$. We know then that $\theta(t)$, the polar angle of this solution, satisfies

$$
\psi(b) \leqq \theta(b) \leqq \omega(b)
$$

and the polar radius satisfies $\rho(b) \geqq R$. Any point $(x, y)$ which satisfies

$$
C x+D y<c_{2}-\delta
$$

lies on the origin side of $C x+D y=c_{2}-\delta$. (Note that $c_{2}-\delta>0$ in the case we are considering.) Hence any points of the sector (2.11) which satisfy (2.12) have $\rho(b)<R$. Thus

$$
C r(b)+D s(b) \geqq c_{2}-\delta .
$$

Let $x_{2}(t), y_{2}(t)$ be a solution of (2.1) with initial conditions $x_{2}(a)=r(a)+x_{1}(a)$ and $y_{2}(a)=s(a)+y_{1}(a)$. This solution satisfies (2.2) and from (2.13)

or

$$
c_{2}-\delta \leqq C\left(x_{2}(b)-x_{1}(b)\right)+D\left(y_{2}(b)-y_{1}(b)\right)=C x_{2}(b)+D_{2}(b)-\delta
$$

$$
C x_{2}(b)+D y_{2}(b) \geqq c_{2} \text {. }
$$


Let $l$ denote the segment of $A x+B y=c_{1}$ between $\left(x_{1}(a), y_{1}(a)\right)$ and $\left(x_{2}(b), y_{2}(b)\right)$. The set of solutions of (2.1) evaluated at $b$ given by

$$
[x(b), y(b) \mid(x(a), y(a)) \in l]
$$

is a connected set (by a simple strengthening of Kneser's theorem, see [12]). Thus there exists an initial condition on $l$, i.e., a point on $l$ such that the solution of (2.1) with these initial values satisfies (2.3).

We indicate now an extension of Theorem 2.1 to the boundary value problem

$$
\begin{aligned}
x^{\prime}=f_{1}(t, x, y), \quad y^{\prime} & =f_{2}(t, x, y), \\
g_{1}(x(a), y(a)) & =c_{1}, \\
g_{2}(x(b), y(b)) & =c_{2} .
\end{aligned}
$$

The $g_{i}(x, y), i=1,2$, are assumed to satisfy a Lipschitz condition like that of the $f_{i}(t, x, y)$, i.e., to satisfy

$$
\begin{array}{ll}
A_{1}(x-y) \leqq g_{1}(x, z)-g_{1}(y, z) \leqq A_{2}(x-y) & \text { if } x \geqq y, \\
B_{1}(x-y) \leqq g_{1}(z, x)-g_{1}(z, y) \leqq B_{2}(x-y) & \text { if } x \geqq y, \\
C_{1}(x-y) \leqq g_{2}(x, z)-g_{2}(y, z) \leqq C_{2}(x-y) & \text { if } x \geqq y, \\
D_{1}(x-y) \leqq g_{2}(z, x)-g_{2}(z, y) \leqq D_{2}(x-y) & \text { if } x \geqq y .
\end{array}
$$

The situation is considerably more complicated than that of Theorem 2.1. Let $G_{1}$ denote the region (possibly null) in the two sectors

$$
\begin{array}{lll}
A_{2} x+B_{2} y \geqq 0, & A_{1} x+B_{1} y \leqq 0, & x \geqq 0, y \geqq 0 ; \\
A_{2} x+B_{1} y \geqq 0, & A_{1} x+B_{2} y \leqq 0, & x \geqq 0, y \leqq 0 .
\end{array}
$$

Let $G_{2}$ denote the union of the following four sectors:

$$
\begin{array}{lll}
C_{2} x+D_{2} y \geqq 0, & C_{1} x+D_{1} y \leqq 0, & x \geqq 0, y \geqq 0 ; \\
C_{2} x+D_{1} y \geqq 0, & C_{1} x+D_{2} y \leqq 0, & x \geqq 0, y \leqq 0 ; \\
C_{1} x+D_{1} y \geqq 0, & C_{2} x+D_{2} y \leqq 0, & x \leqq 0, y \leqq 0 ; \\
C_{1} x+D_{2} y \geqq 0, & C_{2} x+D_{1} y \leqq 0, & x \leqq 0, y \geqq 0 .
\end{array}
$$

It will be required that $G_{1} \cap G_{2}-\{(0,0)\}=\varnothing$. In the case the $g_{i}(x, y)$ are linear this is taken care of in the nonparallel condition on the lines inherent in the definition of $M$. Define

$$
M=\min _{i=1,2 ; j=1,2}\left[p\left(\tan ^{-1}-\frac{A_{i}}{B}, \tan ^{-1}-\frac{C_{i}}{D_{j}}\right), q\left(\tan ^{-1}-\frac{A_{i}}{B_{j}}, \tan ^{-1}-\frac{C_{i}}{D_{j}}\right)\right] .
$$

TheORem 2.3. Let $f_{i}(t, x, y), i=1,2$, be continuous and satisfy (1.7) in $[a, b] \times R$ $\times R$. Let $q_{i}(x, y), i=1,2$, be continuous in $R \times R$, and satisfy (2.16) there. Suppose further that $G_{1} \cap G_{2}-\{(0,0)\}=\varnothing$. If $b-a<M$, there exists at most one solution of (2.1), (2.14), (2.15). 
Proof. We sketch the proof which is essentially that of Theorem 2.1. Suppose there were two solutions $\left(x_{1}(t), y_{1}(t)\right)$ and $\left(x_{2}(t), y_{2}(t)\right)$ and as before let $(\bar{x}(t), \bar{y}(t))$ be the difference of them where $\bar{x}(a)>0$ or $\bar{x}(a)=0$ and $\bar{y}(a)>0$. Then $(\bar{x}(t), \bar{y}(t))$ satisfies

$$
x^{\prime}=F_{1}(t, x, y), \quad y^{\prime}=F_{2}(t, x, y),
$$

where the $F_{i}(t, x, y)$ are as defined in Theorem 2.1. From the Lipschitz conditions (2.16)

$$
\begin{gathered}
A_{2} \bar{x}(a)+\left\{\begin{array}{l}
B_{2} \bar{y}(a) \text { if } \bar{y}(a) \geqq 0 \\
B_{1} \bar{y}(a) \text { if } \bar{y}(a)<0
\end{array}\right\} \geqq 0, \\
A_{1} \bar{x}(a)+\left\{\begin{array}{l}
B_{1} \bar{y}(a) \text { if } \bar{y}(a) \geqq 0 \\
B_{2} \bar{y}(a) \text { if } \bar{y}(a)<0
\end{array}\right\} \leqq 0, \\
\left\{\begin{array}{l}
C_{2} \bar{x}(b) \text { if } \bar{x}(b) \geqq 0 \\
C_{1} \bar{x}(b) \text { if } \bar{x}(b)<0
\end{array}\right\}+\left\{\begin{array}{l}
D_{2} \bar{y}(b) \text { if } \bar{y}(b) \geqq 0 \\
D_{1} \bar{y}(b) \text { if } \bar{y}(b)<0
\end{array}\right\} \geqq 0, \\
\left\{\begin{array}{l}
C_{1} \bar{x}(b) \text { if } \bar{x}(b) \geqq 0 \\
C_{2} \bar{x}(b) \text { if } \bar{x}(b)<0
\end{array}\right\}+\left\{\begin{array}{l}
D_{1} \bar{y}(b) \text { if } \bar{y}(b) \geqq 0 \\
D_{2} \bar{y}(b) \text { if } \bar{y}(b)<0
\end{array}\right\} \leqq 0 .
\end{gathered}
$$

The first two inequalities say that $(\bar{x}(a), \bar{y}(a)) \in G_{1}$ and the last two that $(\bar{x}(b), \bar{y}(b)) \in G_{2}$.

To reach $G_{2},(\bar{x}(t), \bar{y}(t))$ must cross one of the lines bounding $G_{1}$ a last time $t_{1} \geqq a$. Since $(\bar{x}(b), \bar{y}(b)) \in G_{2}$, so $(\bar{x}(t), \bar{y}(t))$ must cross one of the lines bounding $G_{2}$ for a first time $t_{2} \leqq b . M$ is defined so that neither angle $\omega(t)$ or $\psi(t)$ of the comparison equations (2.6) and (2.7) can pass through any angle between a boundary line of $G_{1}$ and one of $G_{2}$. Hence by the Perov Comparison Theorem neither can $\varphi(t)$, the polar angle of the system (2.17).

3. Existence from uniqueness. In this section the boundary value problem

$$
\begin{aligned}
x^{\prime}=f_{1}(t, x, y), \quad y^{\prime} & =f_{2}(t, x, y), \\
g_{1}(x(a), y(a)) & =c_{1}, \\
g_{2}(x(b), y(b)) & =c_{2},
\end{aligned}
$$

will be considered under the hypothesis that there is at most one solution of (3.1), (3.2), (3.3). The object is to establish an existence theorem for suitable curves (3.2) and (3.3).

The first theorems of this sort for two point boundary value problems are due to Lasota and Opial [6] and Jackson [3]. Other results of this kind may be found in [1], [10], [11], [13], [14]. The conditions on $g_{i}(x, y), i=1,2$, will be strengthened as to smoothness over those imposed in $\$ 2$, but there will be no condition of the form (2.16). The major additional assumption is one which relates the $g_{i}(x, y)$ and the $f_{i}(t, x, y)$. We state this as 
(H) If $\left\{\left(x_{n}, y_{n}\right)\right\}$ is a sequence of points in the plane such that $g_{2}\left(x_{n}, y_{n}\right)$ and

$$
\frac{\partial}{\partial x} g_{2}\left(x_{n}, y_{n}\right) f_{1}\left(t_{n}, x_{n}, y_{n}\right)+\frac{\partial g_{2}}{\partial y}\left(x_{n}, y_{n}\right) f_{2}\left(t_{n}, x_{n}, y_{n}\right)
$$

are bounded for bounded $\left\{t_{n}\right\}$, then $\left\{\left(x_{n}, y_{n}\right)\right\}$ lies in a bounded region.

First it needs to be shown that $(\mathrm{H})$ is satisfied in the usually studied case

$$
z^{\prime \prime}+f\left(t, z, z^{\prime}\right)=0, \quad z(a)=A, \quad z(b)=B .
$$

In this case, $g_{1}(x, y)=g_{2}(x, y)=x, f_{1}(t, x, y)=y$, and $f_{2}(t, x, y)=-f(t, x, y)$. $g_{2}\left(x_{n}, y_{n}\right)$ bounded says that $x_{n}$ is bounded. Since $\partial g_{2} / \partial x=1, \partial g_{2} / \partial y=0$, the second condition becomes

$$
1 \cdot y_{n}+0 \cdot f_{2}\left(b, x_{n}, y_{n}\right)=y_{n}
$$

Thus the $\left(x_{n}, y_{n}\right)$ lie in a bounded region and $(\mathrm{H})$ is satisfied. A less trivial example will be given in the next section.

Condition $(\mathrm{H})$ can be equivalently formulated as follows: For any bounded interval $I$,

$$
g_{2}^{2}(x, y)+\left[\operatorname{grad} g_{2}(x, y) \cdot f(x, y)\right]^{2} \rightarrow \infty \quad \text { as } x^{2}+y^{2} \rightarrow \infty
$$

uniformly with respect to $t \in I$, where $f=\left(f_{1}, f_{2}\right)$. (The author is indebted to the referee for this comment and for other suggestions to improve the clarity of presentation.)

TheOREM 3.1. For $i=1,2$, let $f_{i}(t, x, y)$ be continuous on $[a, b+\gamma) \times R \times R, \gamma>0$, and let $g_{i}(x, y)$ be continuously differentiable. Let $g_{1}(x, y)=c_{1}$, when parametrized by arc length, leave every bounded region for sufficiently large arc length in either direction, and let $g_{2}(x, y)=c$ have a real locus for all real c. If $(\mathrm{H})$ holds, if all solutions with initial conditions at $t=a$ exist over $[a, b+\gamma)$, and if there exists at most one solution of (3.1), (3.2) and $g_{2}(x(s), y(s))=c$ for $s \in(b-\varepsilon, b+\varepsilon)$ some $\varepsilon>0$ and all $c$, then there exists at least one (and hence exactly one) solution of (3.1), (3.2), (3.3).

Remarks. The condition that $g_{2}(x, y)=c$ have a real locus for every real $c$ may be replaced by a real locus for $c$ in some open interval provided the conclusion is restricted to $c$ in that interval. A similar theorem is true if the interval $[a, b+\gamma)$ is replaced by $(a-\gamma, b]$ and the uniqueness holds in $(a-\varepsilon, a+\varepsilon)$. That the theorem is not true if uniqueness is not required in a neighborhood has been shown by a counterexample in [6]. Also in [6] an example is given of a second order scalar equation with linear boundary conditions which satisfies all of the hypotheses of Theorem 3.1 except $(\mathrm{H})$ and for which solutions fail to exist.

Proof. Let $P_{0}$ be an arbitrary point of $g_{1}(x, y)=c_{1}$ and suppose that $g_{1}(x, y)=c_{1}$ is parameterized by arc length with $P_{0}$ as initial point. Hereafter we refer to points of this curve by a single real number denoting the parameter. For a fixed $s, a \leqq s \leqq$ 
$b+\varepsilon$, define $T_{s}(P), P \in g_{1}(x, y)=c_{1}$, to be the point set $\{(x(s, P), y(s, P))\}$ where $(x(t, P), y(t, P))$ denotes a solution through $P$ at $t=a$, i.e. $T_{s}(P)$ is the funnel section at $s$ of all solutions through $P$. Since all solutions are continuable, the mapping takes the points of $g_{1}(x, y)=c_{1}$ into nonempty subsets of the plane. On the range of $T_{s}(P)$ define

$$
S\left(T_{s}(P)\right)=\left\{g_{2}(x(s, P), y(s, P)) \mid(x(s, P), y(s, P)) \in T_{s}(P)\right\} .
$$

Let $\Gamma_{s}(P)=S\left(T_{s}(P)\right)$. If the range of $\Gamma_{b}$ is the whole real line, there exists a solution of (3.1), (3.2), (3.3) for every choice of $c_{2}$.

LEMMA 3.1. $\Gamma_{s}(R)$ is connected for $s \in[a, b+\varepsilon)$.

Proof. Since $g_{2}(x, y)$ is continuous $\Gamma_{s}(P)$ will be a compact connected set if $T_{s}(P)$ is. That $T_{s}(P)$ is compact and connected is a consequence of Kneser's theorem and the assumed continuability of solutions. In fact, if $A$ is a compact connected set, $T(A)$ is also. (This is stated in [12] for the autonomous case and can be proved in the nonautonomous case as well, following [4].)

Suppose $\Gamma_{s}(R)=A \cup B$ where $A$ and $B$ are separated. Let $Q_{1} \in A$ and $Q_{2} \in B$. For $i=1,2$ there exists a solution $\left(x_{i}\left(t, P_{i}\right), y\left(t, P_{i}\right)\right)$ such that $P_{i} \in g_{1}(x, y)=c_{1}$, and $g_{2}\left(x_{i}\left(s, P_{i}\right), y_{i}\left(s, P_{i}\right)\right)=Q_{i}$. Let $E$ be the arc of $g_{1}(x, y)=c_{1}$ joining $P_{1}$ and $P_{2}$. In view of the remarks in the preceding paragraph, $\Gamma_{s}(E)$ is connected. Yet $\Gamma_{s}\left(P_{1}\right) \in A$, $\Gamma_{s}\left(P_{2}\right) \in B$, and $A$ and $B$ are separated. This establishes the lemma.

By uniqueness of solutions of boundary value problems, if $s \in(b-\varepsilon, b+\varepsilon)$, $\Gamma_{s}\left(P_{1}\right) \cap \Gamma_{s}\left(P_{2}\right)=\varnothing$. Hence $\Gamma_{s}(P)$ is an interval or a point, and the range of $\Gamma_{s}(R)$ may be ordered in the obvious way.

LeMma 3.2. $\Gamma_{s}$ is monotone for $s \in(b-\varepsilon, b+\varepsilon)$.

Proof. Let $P_{1}<P_{2}<P_{3}$ (ordered by arc length) and suppose $\Gamma\left(P_{2}\right)<\Gamma\left(P_{1}\right)<$ $\Gamma\left(P_{3}\right)$ (the other cases follow similarly). Let $x_{i} \in \Gamma_{s}\left(P_{i}\right), i=1,2,3 . \Gamma_{s}\left(\left[P_{2}, P_{3}\right]\right)$ is a connected set so there exists a point $P^{*}, P_{2} \leqq P^{*} \leqq P_{3}$, such that $x_{1} \in T\left(P^{*}\right)$. Since $P_{1}<P_{2} \leqq P^{*}$ this contradicts uniqueness of solutions of boundary value problems.

Proof of theorem (continued). If the range of $\Gamma_{b}(R)$ is not all of $R$ then $\Gamma_{b}(P)$ is bounded above or below. Suppose $\Gamma_{b}(R)$ is bounded above and let $\eta=\sup \Gamma_{b}(R)$. First of all, it will be established that $\eta \notin \Gamma_{b}(R)$. Suppose that $\eta \in \Gamma_{b}(R)$. Then there exists a solution of (3.1), $(x(t), y(t))$, which satisfies (3.2) and $g_{2}(x(b), y(b))=\eta$. Let $P_{1}=(x(a), y(a))$ (recall $P$ is a parameter denoting arc length on $g_{1}(x, y)=c_{1}$ ). Choose $P_{2}$ and $P_{3}$ such that $P_{2}<P_{1}<P_{3}$. Then, in view of the monotonicity, either $\Gamma_{b}\left(P_{2}\right)>\Gamma_{b}\left(P_{1}\right)$ or $\Gamma_{b}\left(P_{3}\right)>\Gamma_{b}\left(P_{1}\right)$. Since $\eta \in \Gamma_{b}\left(P_{1}\right)$, this contradicts the maximality of $\eta$ and hence $\eta \in \Gamma_{b}(R)$ is impossible.

Since $\eta$ is the sup $\Gamma_{b}(R)$ there exist $z_{n} \in \Gamma_{b}(R)$ such that $z_{n} \uparrow \eta$. To each $z_{n}$ there corresponds a unique point in the $x-y$ plane $P_{n}, P_{n} \in g_{1}(x, y)=c$, and a unique solution of (3.1), $\left(x_{n}(t), y_{n}(t)\right)$ through $P_{n}$ at $t=a$, such that $g_{2}\left(x_{n}(b), y_{n}(b)\right)=z_{n}$. 
Note that Lemma 3.2 guarantees that $P_{n}$ depends monotonely on $n$. Let $\psi_{n}(t)=$ $g_{2}\left(x_{n}(t), y_{n}(t)\right), t \in[a, b+\gamma)$. Then $\psi_{n}(t)$ is differentiable and

$$
\begin{aligned}
\psi_{n}^{\prime}(t)= & \left(\partial g_{2} / \partial x\right)\left(x_{n}(t), y_{n}(t)\right) f_{1}\left(t, x_{n}(t), y_{n}(t)\right) \\
& +\left(\partial g_{2} / \partial y\right)\left(x_{n}(t), y_{n}(t)\right) f_{2}\left(t, x_{n}(t), y_{n}(t)\right)
\end{aligned}
$$

The object of the following argument is to select a convergent subsequence of initial conditions, i.e. points in the plane $\left(x_{n}, y_{n}\right)$. It is an adaptation of an argument originally given by Lasota and Opial [6].

In terms of the parametrization discussed in the beginning of the proof, for fixed $t$ in $(b-\varepsilon, b+\varepsilon), \psi_{n}(t)$ is monotone in $n$ (either increasing or decreasing) since $\left\{P_{n}\right\}$ is monotone in $n$. Further, $\psi_{n}^{\prime}(t) \geqq 0$ or $\psi_{n}^{\prime}(t) \leqq 0$ for infinitely many $n$-for definiteness assume $\psi_{n}(t)$ is monotone increasing in $n$ for $t \in(b-\varepsilon, b+\varepsilon)$ and $\psi_{n}^{\prime}(b) \leqq 0$ for infinitely many $n$. We restrict the argument to these $n$. Let $t^{*} \in$ $[b, b+\varepsilon)$. Then

$$
\frac{\psi_{n}\left(t^{*}\right)-\psi_{n}(b)}{t^{*}-b} \geqq \frac{\psi_{1}\left(t^{*}\right)-\psi_{n}(b)}{t^{*}-b} \geqq K=\min \left[0, \frac{\psi_{1}\left(t^{*}\right)-\eta}{t^{*}-b}\right] .
$$

Let $S_{n}=\left[t \mid t \geqq b ; 0 \geqq \psi_{n}^{\prime}(t) \geqq K\right]$. The sign of $\psi_{n}^{\prime}(b)$, (3.4), and the mean value theorem imply that $S_{n}$ is not empty. Clearly, $S_{n}$ is closed. Let $s_{n}=\min S_{n}$. Then $b \leqq s_{n} \leqq b+\varepsilon$, so there exists a convergent subsequence (which we still label $s_{n}$ ), i.e. $s_{n} \rightarrow s_{0}$. Now $0 \geqq \psi_{n}^{\prime}\left(s_{n}\right) \geqq K$. Note that if $\psi_{n}^{\prime}(b)=0, b=s_{n}$. If $\psi_{n}^{\prime}(b)<0, \psi_{n}(t)<\eta$ until $\psi_{n}^{\prime}(t)=0$ for some $t>b$. This $t$ is in $S_{n}$, hence $\psi_{1}\left(s_{n}\right)<\psi_{n}\left(s_{n}\right)<\eta$. Rewriting the $\psi_{n}\left(s_{n}\right)$ and $\psi_{n}^{\prime}\left(s_{n}\right)$ in terms of $\left(x_{n}\left(s_{n}\right), y_{n}\left(s_{n}\right)\right)$ we are in the situation described by hypothesis $(\mathrm{H})$. Thus $\left\{\left(x_{n}\left(s_{n}\right), y_{n}\left(s_{n}\right)\right)\right\}$ lies in a bounded region of the plane.

Choose a convergent subsequence, i.e. let $s_{n} \rightarrow s_{0}, x_{n}=x_{n}\left(s_{n}\right) \rightarrow x_{0}, y_{n}=$ $y_{n}\left(s_{n}\right) \rightarrow y_{0}$. Let $S_{\delta}$ denote a sphere of radius $\delta$ about $\left(s_{0}, x_{0}, y_{0}\right)$ where $\delta<b+\varepsilon-s_{0}$. For large $n,\left(s_{n}, x_{n}, y_{n}\right) \in S_{\delta}$. Since all solutions exist over $\left[a, s_{0}+\delta\right]$, there exists a $k$ such that $x_{n}^{2}(a)+y_{n}^{2}(a)<k^{2}\left[15\right.$, p. 16]. Let $P_{n}=\left(x_{n}(a), y_{n}(a)\right)$. Let $Q_{1}>P>Q_{2}$ be points of $g_{1}(x, y)=c_{1}$ such that $\left\|Q_{1}\right\|>k$ and $\left\|Q_{2}\right\|>k(\|\cdot\|$ is Euclidean norm). Such points exist by the assumption that $g_{1}(x, y)=c$ does not lie in a bounded region. From the monotonicity of $\Gamma_{b}$ it follows that $\Gamma_{b}\left(Q_{1}\right)>\Gamma_{b}\left(P_{n}\right)$ or $\Gamma_{b}\left(Q_{2}\right)>$ $\Gamma_{b}\left(P_{n}\right)$ for every $n$. Hence either $\Gamma_{b}\left(Q_{1}\right) \geqq \eta$ or $\Gamma_{b}\left(Q_{2}\right) \geqq \eta$. This is a contradiction of the maximality of $\eta$. The other cases are established similarly.

Theorem 3.1 includes the theorem in [11] as a special case (which in turn includes the theorem of this type in [1], [3], [6], [10], [14]).

4. Examples. In this section the theorems of the preceding two sections will be illustrated. Consider the problem

$$
\begin{array}{cl}
x^{\prime}=f_{1}(t, x, y), & y^{\prime}=f_{2}(t, x, y) ; \\
g_{1}(x(a), y(a))=0, & g_{2}(x(b), y(b))=0 .
\end{array}
$$

Suppose $g_{1}(u, v)=u-v, g_{2}(u, v)=u+v+u / 1+u^{2}, g_{1}(u, v)$ is linear, and $g_{2}(u, v)$ is 
linear in $v$, so in (2.13) $A_{1}=A_{2}=1, B_{1}=B_{2}=-1, D_{1}=D_{2}=1$. Further, $\frac{7}{8}(u-w) \leqq$ $g_{2}(u, v)-g_{2}(w, v) \leqq 2(u-w)$ as may be seen from bounding the derivative. Thus the region $G_{1}$ is the line $y=x$ and $G_{2}$ is the closed region between $2 x+y=0$ and $7 x+8 y=0$. (The first and third sectors in the definition of $G_{2}$ are null since both lines have negative slope.) Thus if $f_{i}(t, x, y), i=1,2$, are Lipschitzian, Theorem 2.3 applies. For example, suppose $f_{1}(t, x, y)=\sin y, f_{2}(t, x, y)=x-y$. Then in (1.7), $L_{1}=L_{2}=0, K_{1}=-1, K_{2}=1, M_{1}=M_{2}=1, N_{1}=N_{2}=1$. The comparison systems (2.6) and (2.7) become $u^{\prime}=-v, v^{\prime}=u-v$, and $w^{\prime}=z, z^{\prime}=w-z$. These systems are linear and can be solved explicitly. The computation of $M$, however, is tedious and will be omitted, but if $b-a<M$, the problem has at most one solution.

To show that a solution exists, Theorem 3.1 will be applied. Except for hypothesis (H) all of the conditions of the theorem are satisfied if $b-a<M$. Suppose that $\left(x_{n}, y_{n}\right)$ is a set of points such that the conditions of $(\mathrm{H})$ are satisfied but that the $\left(x_{n}, y_{n}\right)$ do lie in a bounded region. If $\left(x_{n}, y_{n}\right)$ are such that $g_{2}\left(x_{n}, y_{n}\right)$ is bounded, then there exist numbers $c_{1}$ and $c_{2}$ such that $\left(x_{n}, y_{n}\right)$ are in the region between $g_{2}(x, y)=c_{1}$ and $g_{2}(x, y)=c_{2}$. From the form of these curves, i.e. $x+y+x /\left(1+x^{2}\right)$ $=c$, we observe that if $c$ is bounded, then as $x \rightarrow \pm \infty, y \rightarrow \mp \infty$ (and as $y \rightarrow \pm \infty$, $x \rightarrow \mp \infty)$. Since every point $\left(x_{n}, y_{n}\right)$ is on some such curve with $c_{1}<c<c_{2}$, and $\left\{\left(x_{n}, y_{n}\right)\right\}$ does not lie in a bounded region, then either $x_{n} \rightarrow \infty, y_{n} \rightarrow-\infty$, or $x_{n} \rightarrow-\infty$ and $y_{n} \rightarrow \infty$ as $n \rightarrow \infty$. However,

$$
\frac{\partial g_{2}}{\partial x}\left(x_{n}, y_{n}\right) f_{1}\left(t, x_{n}, y_{n}\right)+\frac{\partial g_{2}}{\partial y}\left(x_{n}, y_{n}\right) f_{2}\left(t, x_{n}, y_{n}\right)=\left(1+\frac{1-x_{n}^{2}}{\left(1+x_{n}\right)^{2}}\right) \sin y+x_{n}-y_{n}
$$

is bounded. Since the first term of this expression is bounded, $\left(x_{n}-y_{n}\right)$ must be bounded and this is incompatible with $x_{n}$ and $y_{n}$ being unbounded and (eventually) of opposite sign. Thus $\left(x_{n}, y_{n}\right)$ must lie in a bounded region and $(\mathrm{H})$ holds. Theorem 3.1 then yields an existence theorem.

It is possible to apply Theorem 3.1 without using Theorem 2.3. For example, consider the boundary value problem

$$
\begin{gathered}
x^{\prime}=x+2 y \sin ^{2} x, \quad y^{\prime}=y / 2+\sin ^{2} x ; \\
y(a)=c, \quad x(b)-y^{2}(b)=c_{2} .
\end{gathered}
$$

The conditions of Theorem 3.1 are obviously satisfied except for the existence of at most one solution (continuability, for example, follows from [7, p. 9]). To prove the existence of at most one solution, note first that a line $y=c_{1}$ cuts each curve $x-y^{2}=c$ exactly once. Hence if $\left(x_{1}(t), y_{1}(t)\right)$ and $\left(x_{2}(t), y_{2}(t)\right)$ were two solutions, then labeling may be chosen so that

$$
g_{2}\left(x_{1}(a), y_{1}(a)\right)>g_{2}\left(x_{2}(a), y_{2}(a)\right) .
$$

Let $G_{i}(t)=g_{2}\left(x_{i}(t), y_{i}(t)\right), i=1,2$.

$$
G_{i}^{\prime}(t)=x_{i}(t)+2 y_{i}(t) \sin ^{2} x_{i}(t)-2 y_{i}(t)\left[y_{i}(t) / 2+\sin ^{2} x_{i}(t)\right]=G_{i}(t) .
$$


Hence $G_{i}(t)=G_{i}(a) e^{t}$. Since $G_{1}(a)>G_{2}(a), G_{1}(b)>G_{2}(b)$ contradicting the second boundary condition. Thus there can be at most one solution of the boundary value problem. By Theorem 3.1 there is exactly one solution.

\section{REFERENCES}

1. P. B. Bailey and L. F. Shampine, Existence from uniqueness for two point boundary value problems, J. Math. Anal. Appl. 25 (1969), 569-574. MR 39 \#530.

2. P. B. Bailey, L. F. Shampine and P. Waltman, Nonlinear two point boundary value problems, Math. Sci. and Engineering, vol. 44, Academic Press, New York, 1968. MR 37 \#6524.

3. L. Jackson, Subfunctions and second-order ordinary differential inequalities, Advances in Math. 2 (1968), 307-363. MR 37 \#5462.

4. E. Kamke, Zur Theorie der Systeme gewöhnlicher Differential-Gleichungen. II, Acta Math. 58 (1932), 57-85.

5. M. A. Krasnosel'skiĭ, A. I. Perov, A. I. Povolockiǐ and P. P. Zabreĭko, Plane vector fields, Fizmatgiz, Moscow, 1963; English transl., Academic Press, New York, 1966. MR 31 \#719; MR 34 \#6738.

6. A. Lasota and $\mathrm{Z}$. Opial, On the existence and uniqueness of solutions of a boundury value problem for an ordinary second-order differential equation, Colloq. Math. 18 (1967), 1-5. MR 36 \#2871.

7. V. V. Nemyckir and V. V. Stepanov, Qualitative theory of differential equations, GITTL, Moscow, 1949; English transl., Princeton Math. Series, no. 22, Princeton Univ. Press, Princeton, N. J., 1960. MR 22 \#12258.

8. A. I. Perov, A boundary-value problem for a system of two differential equations, Dokl. Akad. Nauk SSSR 144 (1962), 493-496=Soviet Math. Dokl. 3 (1962), 759-763. MR 27 \#3861.

9. E. Picard, Sur l'application des methods d'approximations successives a l'étude de certaines equations differentielles ordinaires, J. Math. 9 (1893), 217-271.

10. K. Schrader, Existence theorems for second order boundary value problems, J. Differential Equations 5 (1969), 572-584. MR 39 \#532.

11. K. Schrader and P. Waltman, An existence theorem for nonlinear boundary value problems, Proc. Amer. Math. Soc. 21 (1969), 653-656. MR 39 \#533.

12. G. R. Sell, On the fundamental theory of ordinary differential equations, J. Differential Equations 1 (1965), 370-392. MR 31 \#405.

13. L. F. Shampine, Existence and uniqueness for nonlinear boundary value problems, J. Differential Equations 5 (1969), 346-351. MR 39 \#534.

14. P. Waltman, Existence and uniqueness of solutions to a nonlinear boundary value problem, J. Math. Mech. 18 (1968), 585-586. MR 38 \#3504.

15. T. Yoshizawa, Stability theory by Liapunov's second method, Math. Soc. Japan, no. 9, Tokyo, 1966. MR 34 \#7896.

UNIVERSITY OF IOWA, IOWA CrTy, IOWA 52240. 